\title{
The Risk of Contagion Spreading and its Optimal Control in the Economy ${ }^{\dagger}$
}

\author{
Olena Kostylenko $^{1, *}$, Helena Sofia Rodrigues ${ }^{1,2}$, Delfim F. M. Torres ${ }^{1}$ \\ ${ }^{1}$ Center for Research and Development in Mathematics and Applications (CIDMA), Department of Mathematics, University of Aveiro, \\ 3810-193 Aveiro, Portugal \\ ${ }^{2}$ School of Business Studies, Polytechnic Institute of Viana do Castelo, 4930-678 Valença, Portugal
}

\begin{abstract}
The global crisis of 2008 provoked a heightened interest among scientists to study the phenomenon, its propagation and negative consequences. The process of modelling the spread of a virus is commonly used in epidemiology. Conceptually, the spread of a disease among a population is similar to the contagion process in economy. This similarity allows considering the contagion in the world financial system using the same mathematical model of infection spread that is often used in epidemiology. Our research focuses on the dynamic behaviour of contagion spreading in the global financial network. The effect of infection by a systemic spread of risks in the network of national banking systems of countries is tested. An optimal control problem is then formulated to simulate a control that may avoid significant financial losses. The results show that the proposed approach describes well the reality of the world economy, and emphasizes the importance of international relations between countries on the financial stability.
\end{abstract}

Keywords Contagion, Financial Virus, Infection Spreading, Epidemiological Model, Network, Optimal Control.

AMS 2010 subject classifications 49M05, 91G80, 92D30.

DOI: $10.19139 /$ soic.v7i3.833

\section{Introduction}

Formation of the world market without national barriers contributed to the beginning and further development of a fundamentally new global power system, worldwide. This process, that is called globalization, is based on the growing interconnectedness and interdependence of the modern world, which characterizes the development of international relations in all spheres of the world community. This leads to an increase in the role of external factors in the evolution of all the countries participating in the process.

Scientists in the field of economics define the concept of globalization as an increase in the scale of capital movements, currency flows, foreign trade in goods and services, migration of people, and exchange of information, ideas and technologies. The World Bank defines globalization as the "Freedom and ability of individuals and firms to initiate voluntary economic transactions with residents of other countries". The International Monetary Fund (IMF) defines globalization as "The growing economic interdependence of countries worldwide through increasing volume and variety of cross-border transactions in goods and services, freer international capital flows, and more rapid and widespread diffusion of technology" [8]; "The process by which the world becomes a single place" [12]. Economic globalization is the increasing economic interdependence of national economies across the world through a rapid increase in cross-border movement of goods, services, technology, and capital [9]. In this way,

\footnotetext{
${ }^{\dagger}$ This work is part of first author's Ph.D., which is carried out at University of Aveiro under the Doctoral Program in Applied Mathematics MAP-PDMA, of Universities of Minho, Aveiro and Porto.

${ }^{*}$ Correspondence to: Olena Kostylenko (Email: o.kostylenko@ua.pt). Center for Research and Development in Mathematics and Applications (CIDMA), Department of Mathematics, University of Aveiro, 3810-193 Aveiro, Portugal.
}

ISSN 2310-5070 (online) ISSN 2311-004X (print)

Copyright (C) 2019 International Academic Press 
world globalization is a process of uniting the world into a single system of global property, where the global world economy is characterized by a close interconnection of economic development of different countries.

Synchronization of economic relations is constantly felt. However, as economic agents react more strongly to negative shocks than to positive ones, synchronization becomes more noticeable during a crisis. This is also facilitated by mass media, as they instantly make information about shocks available to a wide range of users.

The global crisis of 2008 has become a significant source of knowledge about the process of synchronization of economic relations during the crisis. It reveals that economically weakly connected countries in stable periods, demonstrate a unidirectional movement of macro indicators during the crisis period. This happens because the crisis in one country provokes a crisis in another(s). This effect is termed financial contagion and it is capable of generating a global network epidemic. Therefore, nowadays the prevention of the global economic crisis and risk minimization are important tasks in the field of economics.

The definition of contagion, which scientists consider in their works, varies considerably. However, there is a similarity in all considered models: they always attempt to provide a structure, explaining why a shock in one country may be transmitted elsewhere [10]. Mechanisms of mutual influence of countries in crisis situations are studied using mathematical and instrumental methods of economic analysis. The main stage of modelling the epidemic of the financial crisis is the choice of a mathematical model that adequately describes the process.

Several researchers draw an analogy between economic and natural viruses, between network and biological epidemics. From a mathematical point of view, the process of spreading a financial virus in the economy is similar to the spread of a viral disease. Therefore, these processes are described by similar models of differential equations. Because economic interactions of countries form a network, the use of network theory can enrich our understanding of financial systems as complex systems, which is used to explain the process of virus contamination in the network.

Here, we simulate an epidemiological process of financial contagion using the Kermack and McKendrick SIR model [11]. The process of infection spreading among the agents is studied through network interconnections. Then, similarly to [13], we apply optimal control theory to minimize the negative consequences of the spread of the virus.

The remainder of this paper is divided into five sections. Section 2 provides a detailed description of the data employed. Section 3 presents the proposed epidemiological model for investigating the behaviour of the contagion spreading and Section 4 shows how the spread of infection occurs in the network. Section 5 details the optimal control problem and discusses policy implications. Finally, Section 6 summarizes and concludes our study.

\section{Data}

The complex study of country interrelations shows which national banking systems are most exposed to a particular country, both on an immediate counterparty basis and on an ultimate risk basis. Claims on an immediate counterparty basis capture lending to a borrower that resides in the counterparty country, while claims on an ultimate risk basis capture lending to a borrower in any country that is guaranteed by an entity that resides in the counterparty country [2]. We use the Bank for International Settlements (BIS) Consolidated Banking Statistics database (consolidated foreign claims of reporting banks - ultimate risks basis) to extract data from Table B4 "Residence of counterparty, by nationality of reporting bank" of [4]. Table B4 of [4] provides the total claims on an ultimate risk basis by nationality of reporting bank. The country of ultimate risk or where the final risk lies is defined as the country in which the guarantor of a financial claim resides and/or the country in which the head office of a legally dependent branch is located [3].

For our research, we have chosen 13 (thirteen) European and Non-European developed countries (Portugal, Italy, Spain, Ireland, Japan, Belgium, France, United Kingdom, Austria, United States, Sweden, Canada, Germany), based on statistical data from BIS for the end of 2017. 


\section{Epidemiological model}

There are many mathematical models (SIS, SIR, SEIR, MSIR, ...) that are widely used for modelling infectious diseases [1, 14]. These epidemiological models are based on dividing the population into compartments, with the assumption that every individual in the same compartment has the same characteristics $[5,15,17]$. In this paper, we use the classic Kermack and McKendrick SIR model [11] to describe the epidemic spreading. This model is a three-state model that describes the proportion of a population that is infected, susceptible to infection and recovered after a disease; and assumes that individuals that leave one class must enter another [7]. We assume that contagion can be transmitted from a country with an infected financial system to another one, which has not yet been infected and, after recovery, the country produces immunity for a long time.

Mathematically, the SIR model is described by the following system of ordinary differential equations that govern its laws of motion:

$$
\left\{\begin{array}{l}
\frac{d S(t)}{d t}=-\beta S(t) I(t), \\
\frac{d I(t)}{d t}=\beta S(t) I(t)-\gamma I(t), \\
\frac{d R(t)}{d t}=\gamma I(t),
\end{array}\right.
$$

$t \in[0, T]$, subject to the initial conditions

$$
S(0)=S_{0}>0, \quad I(0)=I_{0}>0, \quad R(0)=R_{0} \geq 0 .
$$

The total population size $N=S(t)+I(t)+R(t)$ keeps constant for $t \in[0, T]$. Susceptible individuals $(S)$ can become infected with the disease, and then move into the infected class. Infected individuals $(I)$ infect susceptible individuals and continue to exist in the infected class before moving into the recovered class. Recovered individuals $(R)$ are no longer infectious and have immunity [7].

Parameters $\beta$ and $\gamma$ are, respectively, the transmission rate, that is, the probability of the spread of infection, and the recovery rate.

Imposing economic sense to the epidemiological model, we consider the following assumptions:

- initially, only one country is contagious and no one is recovered: $S(0)=12, I(0)=1$, and $R(0)=0$;

- a contagious country $I$ can infect a susceptible country $S$ through their interconnections if the last one has not enough money in reserve to cover possible risk losses;

- an infected counterparty country has all its foreign claims infected;

- the possibility of infection is higher when there are more outstanding debts in the total amount of debts;

- the possibility that a country performs its obligations as a borrower depends on the country's credit rating since it takes into account not only countries' debt but also assets.

Based on the above assumptions, the infection spreading rate and the recovery rate are calculated as follows:

$$
\begin{gathered}
\beta_{i}=\frac{\sum_{j=1}^{13} a_{i j}}{\sum_{i=1}^{13} \sum_{j=1}^{13} a_{i j}}, \quad i \in\{1, \ldots, 13\}, \\
\gamma_{i}=\frac{1}{101-C_{i}}, \quad i \in\{1, \ldots, 13\},
\end{gathered}
$$

where the exposure $a_{i j}$ is calculated using the data available from BIS and the credit rating $C_{i}$ is taken from Trading Economics [19]. The computed values of contagion spreading rate and the speed of recovery are given in Figure 1. 


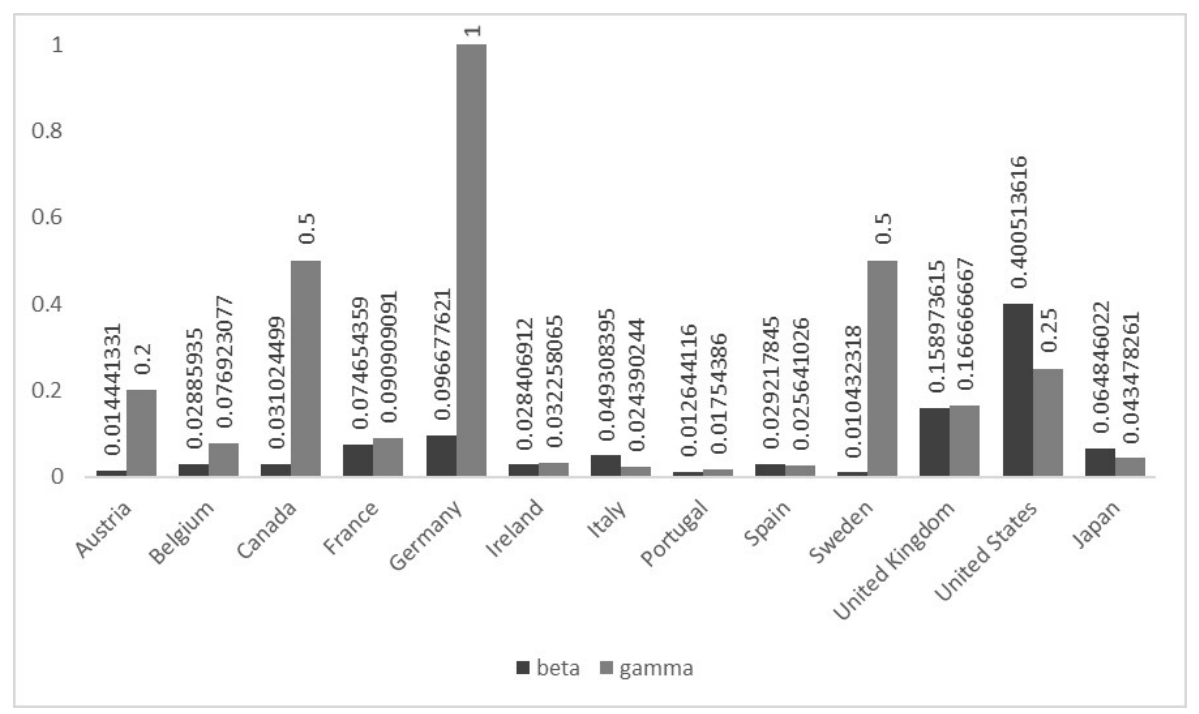

Figure 1. Summary statistics of $\beta$ and $\gamma$ parameters for the 13 European and Non-European developed countries considered in our study.

The model (1)-(2) describes that the contagion begins with a country that spreads a disease by infecting its neighbours, where the number of new infectious countries generated by the initial one is called the reproduction rate. The reproduction rate $\mathcal{R}_{0}=\frac{\beta}{\gamma}$ is greater than 1 when an infected country, on average, spreads it to at least one additional country, that will then do the same thing, and so on. It leads to an epidemic, with the number of countries, that receive contagion, growing exponentially. In contrast, contagion is generally considered to fail when $\mathcal{R}_{0}$ is less than 1.

By solving the initial value problem (1)-(2), we obtain results according to which the epidemiological model shows a different behaviour of contamination, depending on the values of the $\beta$ and $\gamma$ parameters of the first originally infected country.

All countries in our sample can be divided into 4 groups, depending on the value of $\gamma$ and $\mathcal{R}_{0}$ : see Table 1 . For

Table 1. Grouping of the 13 considered countries, depending on the $\gamma$ parameter and $\mathcal{R}_{0}$.

\begin{tabular}{cccc}
\hline Group 1 & Group 2 & Group 3 & $\begin{array}{c}\text { Group 4 } \\
\gamma<0.5\end{array}$ \\
& $0.5 \leq \gamma<0.1$ & $\begin{array}{c}0.1<\gamma \leq 1 \\
\mathcal{R}_{0}>0.9\end{array}$ & $\mathcal{R}_{0}<0.9$ \\
\hline PT & FR & USA & CA \\
IT & BE & GB & AT \\
ES & & & SE \\
IE & & & DE \\
JP & & & \\
\hline
\end{tabular}


illustration purposes, we present four different scenarios of contagion spreading, depending on the group to which the initially infected country belongs: see Figure 2.
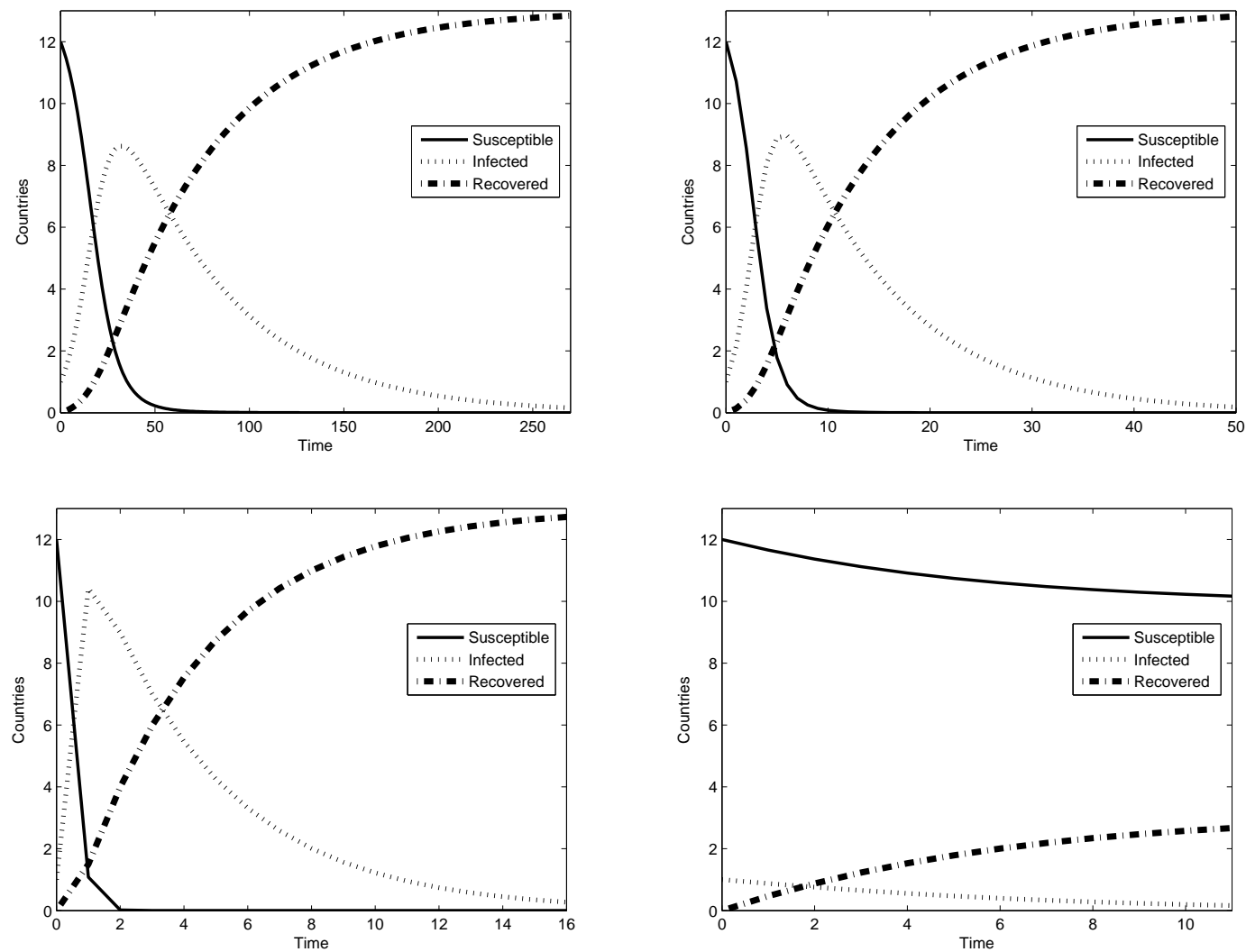

Figure 2. The SIR contagion risk model. From upper-left to lower-right: Portugal, France, USA and Canada as initially infected country.

The first scenario is when the infection begins in Portugal. The results demonstrate that in the case where Portugal is the starting point for the spread of financial contamination, this process takes a long time, the infection reaches its peak in about 3 years, and the process of recovery also occurs very slowly.

The second scenario is when the initially infected country is France. Having a higher risk of infection than Portugal, but significantly higher credit rating than the Portuguese financial system, the infection reaches a contagion-free equilibrium $(I(T)=0)$ five times faster than in previous scenario.

For the third scenario, we chose the United States of America (USA). As for the USA, the $\beta$ parameter is higher than $\gamma$, that is, the contamination rate is higher than recovery. This translates in the shortest period of time the largest number of countries become infected.

Our fourth scenario has Canada as the initially infected country. The situation with Canada is the most optimistic. Indeed, the system reaches its equilibrium in a slightly shorter period of time than with the USA and, simultaneously, there is a minimum number of countries affected because $\mathcal{R}_{0}<1$.

In next sections of network analysis (Section 4) and optimal control (Section 5), we consider only the problematic situations, that is, the cases when $\mathcal{R}_{0}>1$ : the results of simulations for contagion spreading from Canada are not shown because in this situation there is no need to take control measures. 


\section{Network}

To visualize the spread of infection among participants in our sample, a network analysis was carried out. Countries, according to graph theory, are represented in the form of nodes (vertices) while edges (links) mean the existence of financial ties between the countries. According to the data from BIS (Table B4 of [4]), all $n=13$ countries that were chosen for our sample are connected to each other, but none with themselves. In other words, each vertex has the same number of neighbours and the network of countries' interrelations, for our sample, can be represented as a complete graph $K_{n}$, where each vertex has the same degree $n-1$ and the graph has $n(n-1) / 2$ edges. According to these characteristics, the network topology in our research was chosen as a fully connected network.

The dynamics of contagion propagation in the network was investigated using the NetLogo multi-agent programmable modeling environment [20]. The results are shown in Figure 3 for the three endemic scenarios.
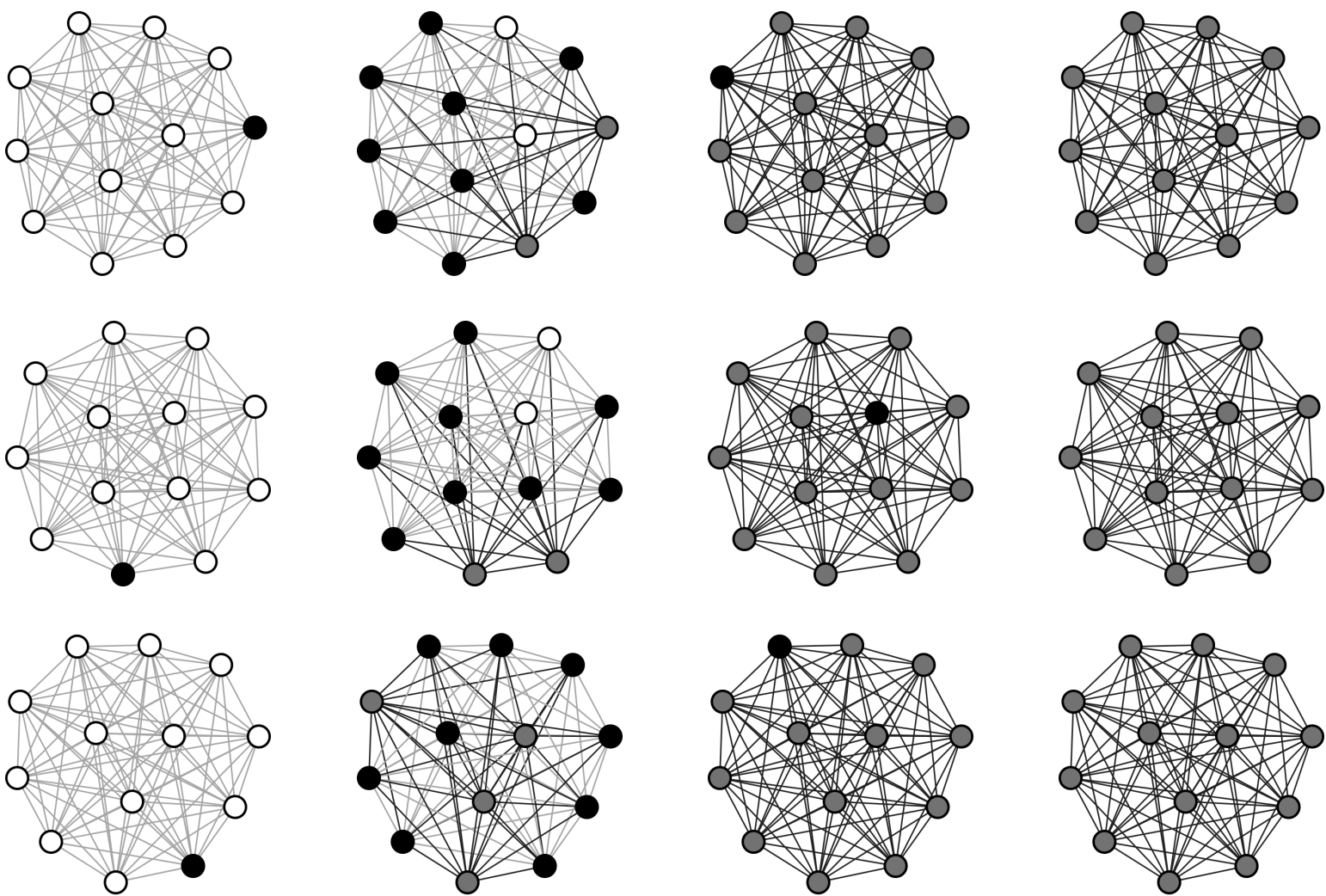

Figure 3. Virus spreading in the network of 13 countries with parameters $\beta$ and $\gamma$ as in Figure 1. From upper-row to lower-row: Portugal ( $T=0, T=35, T=162, T=270$ months), France ( $T=0, T=5, T=49, T=50$ months) and USA ( $T=0, T=2, T=15, T=16$ months).

Each node in the network represents a random country from our sample. A white node represents a susceptible country, black an infected country. The first column from Figure 3 represents the initial moment $\left(T_{0}\right)$, where only one node has an infection, while others are susceptible. In each time step, an infected node attempts to infect all of its susceptible neighbours. The recovered nodes, represented in grey, cannot be infected. Susceptible neighbours will get an infection and change their colour to black with probability $\beta$. Infected nodes will be recovered and change their colour to grey with probability $\gamma$. When a node becomes recovered, the links between it and its 
neighbours change their colour to grey and are no longer possible vectors for contagion spreading [18]. The results we obtain here are in agreement with those of Section 3 (compare Figures 2 and 3).

\section{Optimal control}

All financial institutes need to be under financial supervision in order to prevent contagion spreading and avoid serious consequences. Such a role, in each state, is carried out by the Central Bank, which acts as a supervisory competent authority. Since one of the main goals is to prevent the global spread of infection, we pose the following optimal control problem in Bolza form: functional

$$
\min J[I(\cdot), u(\cdot)]=I(T)+\int_{0}^{T} b u^{2}(t) d t
$$

subject to the control system

$$
\left\{\begin{array}{l}
\frac{d S(t)}{d t}=-\beta S(t) I(t), \\
\frac{d I(t)}{d t}=\beta S(t) I(t)-\gamma I(t)-u(t) I(t), \\
\frac{d R(t)}{d t}=\gamma I(t)+u(t) I(t),
\end{array}\right.
$$

where $S(0)=S_{0}, I(0)=I_{0}, R(0)=R_{0}, b>0, u(t) \in[0,1]$. The integral in the objective functional (5) means a general cost of financial assistance, necessary to prevent the spread of contagion and economic decline in the period $[0, T][13]$. The control functions $u(\cdot)$ are selected within a special set of admissible controls. It consists of those functions $u=u(t)$, which are piecewise continuous functions on the interval $[0, T]$ with values in $U=[0,1]$. The control $u(t)$ denotes the rate at which assistance will be provided to a contagious country, that is, it is the ratio between the financial support from the Central Bank at time $t$ and the financing needed by the countries at that time. If the Central Bank provides a full support at time $t$, covering all necessary risks, then $u(t)=1$. If the country receives no financial lending at $t$, then one has the control $u(t)=0$. The smaller the value of the optimality criterion $J[I(\cdot), u(\cdot)]$, the more beneficial the control $u$ is. In our simulations, the cost of control measures $b$ is taken to be 1.5 , motivated by the value of possible recapitalization with state funds considered in [16].

The optimal control problem in Bolza form (5)-(6) was rewritten in the following equivalent Mayer form and solved in the BOCOP optimal control solver [6]:

$$
\min J[S(\cdot), R(\cdot), Y(\cdot)]=N-S(T)-R(T)+Y(T)
$$

subject to

$$
\left\{\begin{array}{l}
\frac{d S(t)}{d t}=\beta S^{2}(t)+\beta S(t)(R(t)-N), \\
\frac{d R(t)}{d t}=\gamma(N-S(t)-R(t))+u(t)(N-S(t)-R(t)), \\
\frac{d Y(t)}{d t}=b u^{2}(t), \\
S(0)=S_{0}, \quad R(0)=0, \quad Y(0)=0, \\
u(t) \in[0,1] .
\end{array}\right.
$$

The simulation results are shown in Figures 4-6. They consist of the behavioural curves of our SIR contagion risk model and demonstrate the behaviour of the curves with and without optimal control, for different scenarios. 
Figure 4 shows the behaviour of the curves before applying the optimal control and after, for the first scenario, when the infection begins in Portugal. Comparison of the graphics shows that the process of damping of the infection will occur almost twice as fast if optimal control is applied, that is, the recovery process for countries after financial contamination will occur faster if they receive financial support in an optimal way.

The results for the second scenario, that are presented in Figure 5, confirm the similarity of the conclusions that were formulated for the first scenario. The infection process will slow down and, simultaneously, the recovery accelerates. Moreover, the application of optimal control helps more countries to remain as susceptible.

Figure 6 shows the behaviour of the curves for the third scenario. We see that the force of infection is quite high and that the application of optimal control slightly helps to accelerate the recovery process, which leads to a damping of infection.
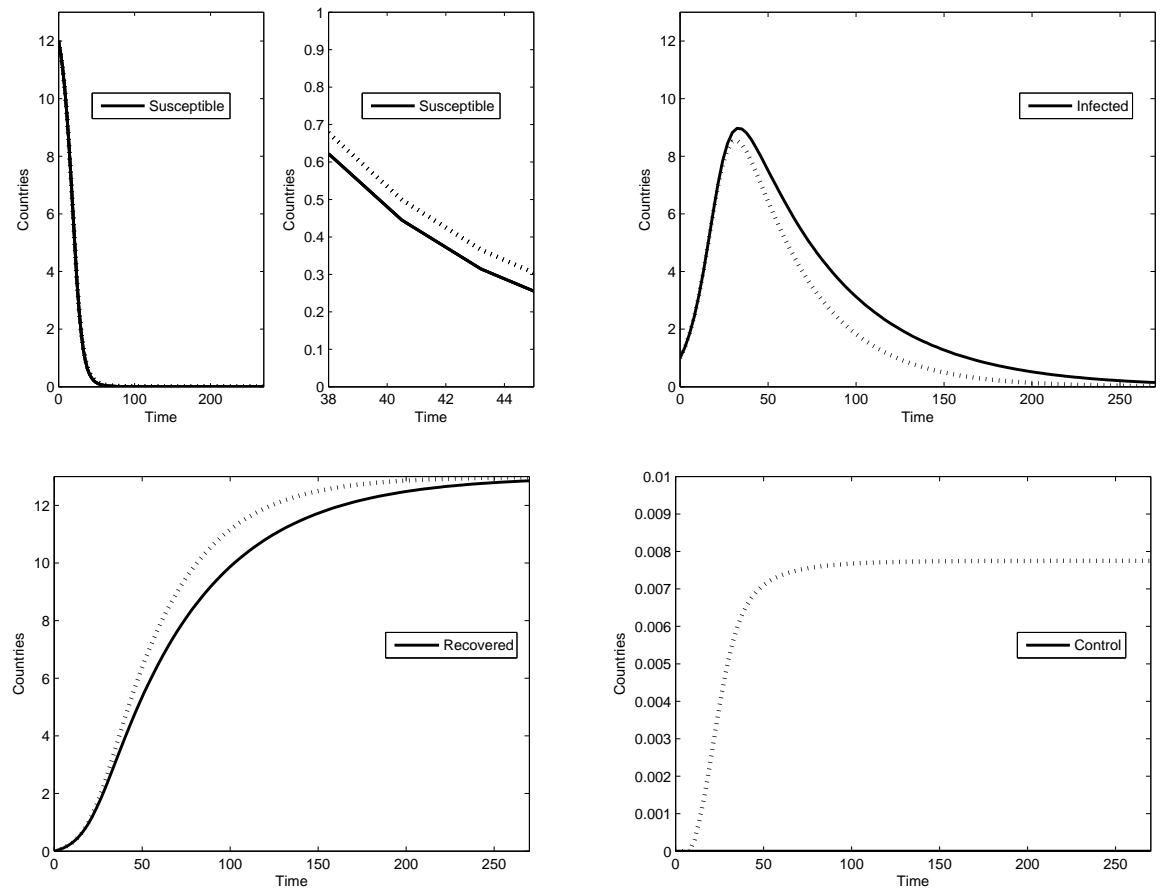

Figure 4. Contagion risk from Portugal (Scenario 1): with optimal control (dotted line) vs without control (continuous line).

\section{Conclusion}

We investigated the process of financial contamination from the point of view of infection spreading among countries that form an interconnected closed-form system. It was shown how strongly the behaviour of infection depends on the values of the transmission and recovery rates, respectively parameters $\beta$ and $\gamma$. Interestingly enough, the results obtained by the continuous-time epidemiological model (1), which are usually valid for a large $N$ (large population), are in agreement with those obtained by the discrete-time (network) epidemiological model of Section 4, which are usually used for a small population $N$. The application of optimal control has justified itself, showing how significant and successful the results can be on the way to recovery, thus demonstrating the need for a thorough control of the quantity guaranteed loans and the financial stability of countries in order to avoid the negative effects of financial risks. The obtained infection-recovery process corroborates all sorts of macroeconomic bibliography premises and therefore validates existing literature within economical-cycle expectations. 

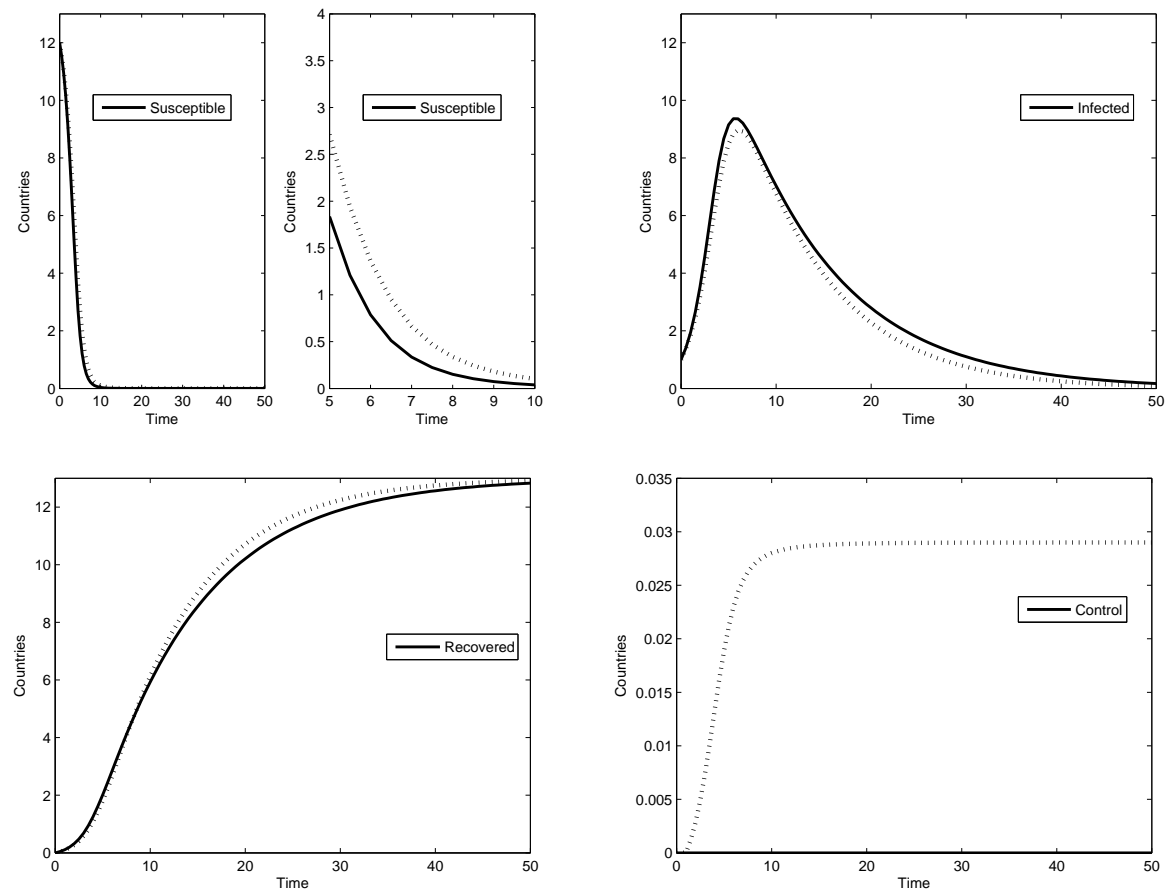

Figure 5. Contagion risk from France (Scenario 2): with optimal control (dotted line) vs without control (continuous line).
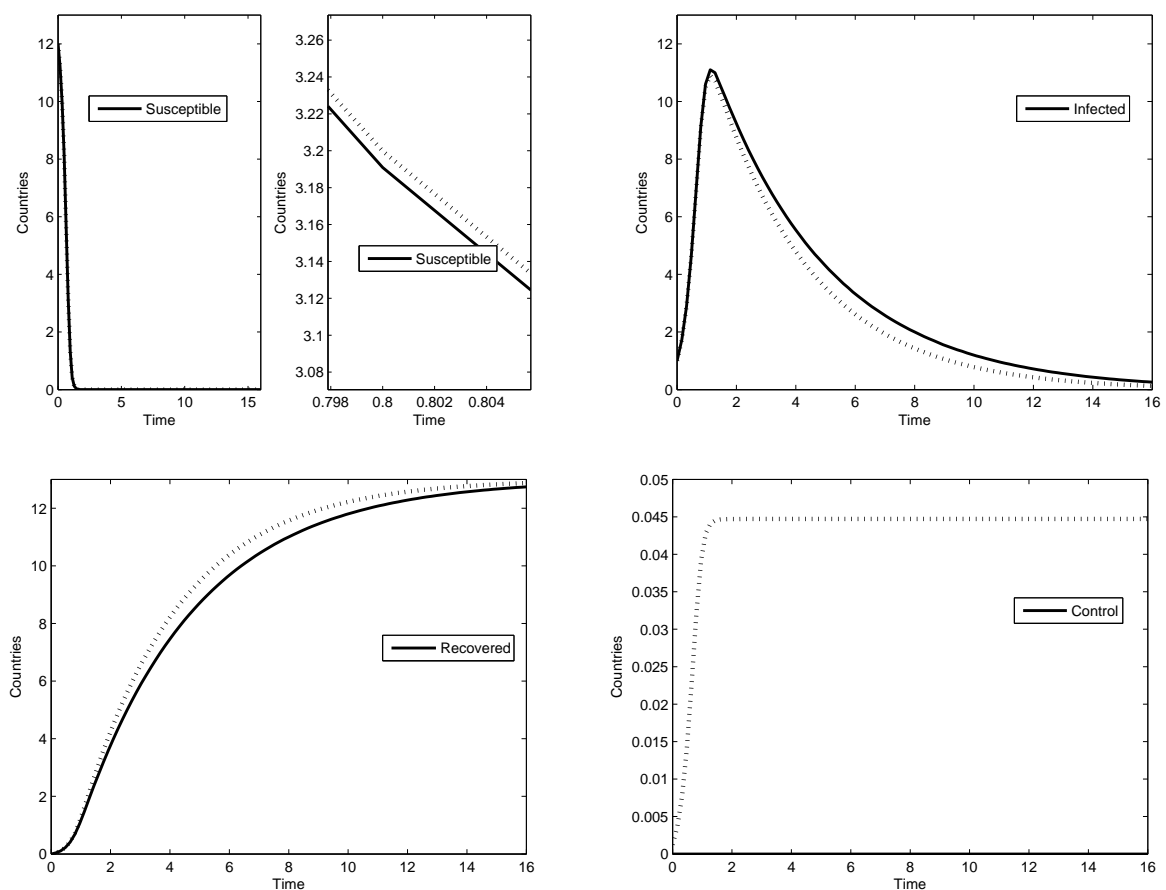

Figure 6. Contagion risk from USA (Scenario 3): with optimal control (dotted line) vs without control (continuous line). 


\section{Acknowledgement}

This research was supported by the Portuguese Foundation for Science and Technology (FCT) within project UID/MAT/04106/2019 (CIDMA). Olena Kostylenko was also supported by the Ph.D. fellowship $\mathrm{PD} / \mathrm{BD} / 114188 / 2016$. The authors would like to thank two reviewers for their critical remarks and precious suggestions, which helped them to improve the quality and clarity of the manuscript.

\section{REFERENCES}

1. I. Area, F. Ndaïrou, J. J. Nieto, C. J. Silva and D. F. M. Torres, Ebola model and optimal control with vaccination constraints, J. Ind. Manag. Optim. 14 (2018), no. 2, 427-446. arXiv: 1703.01368

2. BIS, Bank for International Settlements, FAQs, https://www.bis.org/statistics/statstools/fags.htm?m

3. BIS, Bank for International Settlements, Guidelines to the international consolidated banking statistics, Monetary and Economic Department, Bank for International Settlements Press \& Communications, Basel, Switzerland, 2008.

4. BIS, Bank for International Settlements, Consolidated banking statistics, $19 \quad$ July 2018 https://www.bis.org/statistics/consstats.htm?m

5. J. C. Blackwood and L. M. Childs, An introduction to compartmental modeling for the budding infectious disease modeler, Lett. Biomath. 5 (2018), no. 1, 195-221.

6. BOCOP, An open source toolbox for optimal control, Team Commands, Inria Saclay, 2017. http: / / bocop. org,

7. M. Haran, An introduction to models for disease dynamics, Department of Statistics, Penn State University, Spatial Epidemiology, SAMSI, 2009. https://www.unc.edu/ rls/s940/samsidisdyntut.pdf

8. IMF, International Monetary Fund, World Economic Outlook, 1997.

9. R. M. Joshi, International Business, Oxford University Press, New Delhi and New York, 2009.

10. G. L. Kaminsky and C. M. Reinhart, On crises, contagion, and confusion, Journal of International Economics 51 (2000), 145-168.

11. W. O. Kermack and A. G. McKendrick, A contribution to the mathematical theory of epidemics, Proc. Royal Society A 115 (1927), no. 772, 700-721.

12. A. D. King, Culture, globalization and the world-system: Contemporary conditions for the representation of identity, University of Minnesota Press, 1997.

13. O. Kostylenko, H. S. Rodrigues and D. F. M. Torres, Banking risk as an epidemiological model: An optimal control approach, Springer Proceedings in Mathematics \& Statistics 223 (2018), Springer, Cham, 165-176. arXiv: 1707.03500

14. A. P. Lemos-Paião, C. J. Silva and D. F. M. Torres, An epidemic model for cholera with optimal control treatment, J. Comput. Appl. Math. 318 (2017), 168-180. arXiv: 1611.02195

15. J. P. Mateus, P. Rebelo, S. Rosa, C. M. Silva and D. F. M. Torres, Optimal control of non-autonomous SEIRS models with vaccination and treatment, Discrete Contin. Dyn. Syst. Ser. S 11 (2018), no. 6, 1179-1199. arXiv: 1706.06843

16. D. Philippas, Y. Koutelidakis and A. Leontitsis, Insights into European interbank network contagion, Managerial Finance 41 (2015), no. $8,754-772$.

17. A. Rachah and D. F. M. Torres, Analysis, simulation and optimal control of a SEIR model for Ebola virus with demographic effects, Commun. Fac. Sci. Univ. Ank. Sér. A1 Math. Stat. 67 (2018), no. 1, 179-197. arXiv: 1705.01079

18. F. Stonedahl and U. Wilensky, NetLogo virus on a network model, Center for Connected Learning and Computer-Based Modeling, Northwestern University, Evanston, IL, 2008. http://ccl. northwestern. edu/netlogo/models/VirusonaNetwork

19. Trading Economics, Credit Rating, https://tradingeconomics.com/country-list/rating

20. U. Wilensky, NetLogo, Center for Connected Learning and Computer-Based Modeling, Northwestern University. Evanston, IL, 1999. http://ccl.northwestern.edu/netlogo 